\title{
Prevalence of Depression, Anxiety and Cognitive Impairment in Patients with Type 2 Diabetes from the Central Part of Romania
}

\author{
Cernea Simona ${ }^{1,2^{*}}$, Zoltai Cristian ${ }^{3}$, Berbecilă Daniela ${ }^{4}$, Șular Floredana-Laura ${ }^{5,6}$ \\ 1 Department M3/Internal Medicine IV, University of Medicine and Pharmacy, Tîrgu Mureș, Romania \\ 2 Diabetes, Nutrition and Metabolic Diseases Outpatient Unit, Emergency County Clinical Hospital, Tîrgu Mureș, Romania \\ 31 st Psychiatry Clinic, County Clinical Hospital, Tîrgu Mureș, Romania \\ 4 General Medicine, University of Medicine and Pharmacy, Tîrgu Mureș, Romania \\ ${ }^{5}$ Central Laboratory, Emergency County Clinical Hospital, Tîrgu Mureș, Romania \\ ${ }^{6}$ Department M2/Laboratory Medicine, University of Medicine and Pharmacy, Tîrgu Mureș, Romania
}

\begin{abstract}
Objective: The aim of this study was to assess the prevalence of depression, anxiety and cognitive impairment in patients with type 2 diabetes (T2D). Material and methods: We conducted a cross-sectional study in patients with T2D. Depression and anxiety were assessed by questionnaires (PHQ-9, CES-D and GAD-7 respectively), cognitive function by the MoCA test. Additionally, 503 patients' clinic charts were separately analyzed in order to compare the data recorded in the charts with that resulted from the active assessment. Results: In the screening study 216 subjects with T2D were included (62.2 \pm 7.8 years old). 34.3\% of them had depression and $7.4 \%$ presented major depression. 44.9\% of patients with T2D had anxiety (9.2\% major anxiety) and this was highly correlated with depression (OR: 21.139, 95\%Cl: 9.76745.751; $\mathrm{p}<0.0001)$. Women had significantly higher prevalence of depression and anxiety compared to men $(42.1 \%$ vs. $21.7 \%$; $p: 0.0021$ and $51.1 \%$ vs. 34.9\%; p: 0.02), but severe depression was similar between genders (9.0\% vs. 4.8\%; p: 0.29). Significantly more patients had depression and anxiety than recorded in their charts (34.3\% vs. $13.9 \%$ and $44.9 \%$ vs. $9.3 \%$,respectively; $p<0.0001$ for both). $69.0 \%$ of T2D patients had mild, $6.0 \%$ had moderate and none had severe cognitive dysfunction, respectively. Significantly more patients with depression and anxiety had mild and moderate cognitive impairment (p: 0.03 and p: 0.04, respectively). Conclusions: Patients with T2D had a high prevalence of comorbid depression, anxiety and cognitive impairment. Depression and anxiety were significantly more frequent in women. These conditions were under-evaluated and/or under-reported.
\end{abstract}

Keywords: type 2 diabetes, depression, anxiety, cognitive impairment,prevalence

Received: 21 January 2016 / Accepted: 10 March 2016

\section{Introduction}

Diabetes and depression are both chronic conditions with high prevalence worldwide that negatively impact the quality of life of individuals suffering from them (1). When the two diseases are associated, the consequences on health outcomes are very serious. The odds of diabetes chronic complications and functional disabilities are significantly greater, as well as the risk of all-cause mortality: two studies indicated a $36 \%$ to $54 \%$ greater mortality risk in subjects with diabetes and co-morbid depression, while a third showed a hazard ratio of 2.5 higher than in individuals without either diabetes or depression (1-4). In addition, the concomitant presence of depression contributes to decreased self-care and adherence to therapy and higher health-care costs, as well as higher risk for cognitive dysfunction and dementia in patients with diabetes $(1,5,6)$.

Therefore, there is a high interest regarding the association between diabetes and depression and cognitive impairment, respectively $(5,7)$. Data in the literature seem to indicate an increased prevalence of depression in adults with type 2 diabetes (T2D), which is generally considered twice higher than in subjects without T2D, women be-

${ }^{*}$ Correspondence to: Simona Cernea

E-mail: simonacernea@yahoo.com ing more affected than men (8). However, there are very large variations in reported prevalence, from about $10 \%$ to about $48 \%(9-14)$. These might result from ethnic/geographical differences (developed vs. developing nations), type of study (controlled/uncontrolled) or possibly from methods of estimation (database research or active screening) $(1,8)$. In addition, some studies seem to suggest that up to half of subjects with diabetes have undiagnosed depression and therefore are not properly treated, which of course, has important health consequences (15).

Data regarding the prevalence of depression and major depression, respectively as well as of anxiety and cognitive impairment in subjects with T2D in Romania is scarce. A study performed in patients with both type 1 diabetes (T1D) and T2D with poor metabolic control reported that $58 \%$ of them had significant elevated symptoms of depression, but data was not presented separately for patients with T2D (16).

The aim of this study was to assess the prevalence of depression, anxiety and cognitive dysfunction in subjects with T2D from the Central part of Romania. The hypothesis was that the prevalence of depression and of the other two psychiatric conditions is increased and also higher than acknowledged and recorded in patients' charts, as these conditions are not routinely evaluated in clinical practice. 


\section{Material and methods}

This cross-sectional study consisted in an active screening for depression, anxiety and cognitive impairment by specific questionnaires. For this purpose we randomly recruited subjects that came for regular diabetes visits to the Diabetes, Nutrition and Metabolic Diseases Outpatient Unit of the Emergency County Clinical Hospital in Targu Mures in 2015. Every fourth patient that met both inclusion and exclusion criteria was invited to participate in the study. If one patient declined to participate and/ or did not meet inclusion/exclusion criteria, the next one on the list was invited, a.s.o. The study was approved by the Ethics Committees of the Emergency County Clinical Hospital of Targu Mures and of the University of Medicine and Pharmacy of Targu Mures and the patients signed an informed consent before participating in the study.

Patients were included if they were $\geq 30$ years old, with diagnosis of T2D, had at least minimal literacy (able to read and write) and were fluent in Romanian. Exclusion criteria were diagnosis of T1D, secondary diabetes, gestational diabetes, severe diseases (such as, but not exclusively: severe autoimmune diseases or cancer diagnosed less than 5 years). Diagnosis of diabetes was established according to the American Diabetes Association (ADA) criteria (17).

First, a general questionnaire was applied which asked general demographic and medical data and if the patients were ever told they had depression and/or were ever treated for depression. We have decided to assess the depression status by two commonly used and well validated questionnaires: Patient Health Questionnaires-9 (PHQ-9) and Center for Epidemiologic Studies-Depression (CES-D) (18-20). In order to exclude the false positive cases, we decided to consider a patient positive for depression if he/she reached the preset scores in both questionnaires. For CES$\mathrm{D}$, a score $\geq 16$ was considered indicative of mild depression and $\geq 27$ of major depression $(21,22)$. For PHQ-9, a score $\geq 5$ indicated mild depression, $\geq 10$ moderate depression and $\geq 15$ severe depression (23). For the same rationale, severe depression was considered if patients scored both $\geq 15$ on PHQ- 9 and $\geq 27$ on CES-D questionnaire. In addition, the patients filled out the Generalized Anxiety Disorder-7 (GAD-7) questionnaire, as a screening tool for generalized anxiety, for which a score $\geq 5,10$ and 15 , respectively represented cut-off values for mild, moderate and severe anxiety (24). The patients were given instructions and then filled out all questionnaires independently.

In order to evaluate the cognitive status, the patients were administered the Romanian version of the Montreal Cognitive Assessment (MoCA) test. The permission to use the questionnaire was kindly obtained from Kathleen Gallant, MSOT, on behalf of Dr Ziad Nasreddine, MoCA(C Copyright Owner. This is a cognitive screening tests rated on a 30-point scale and a score $\geq 26$ is considered normal, 17-25 points are indicative of mild cognitive impairment, 10-16 points are indicative of moderate cognitive impairment, while $<10$ of severe cognitive impairment (25).
Additional relevant clinical information was collected from the patients' medical charts, including the diagnosed and recorded depression, anxiety and cognitive impairment. The information regarding the three psychiatric conditions were collected from the diabetology medical charts of the patients. The information was available either if the patient directly reported these conditions to their diabetologist, brought a copy of a medical report from a psychiatrist/neurologist or if these diagnoses were mentioned in a hospital discharge letter.

In addition, we separately analyzed retrospectively 320 consecutive charts of patients diagnosed with T2D, registered and regularly seen in the Diabetes, Nutrition and Metabolic Diseases Outpatient Unit of the Emergency County Clinical Hospital in Targu Mures between 20092015, in order to better assess the prevalence of diagnosed/ reported and recorded depression, anxiety and cognitive impairment.

Descriptive statistics was performed for all variables and expressed as mean \pm SD for continuous variables with normal distribution, median (min-max) for non-normally distributed continuous variables and frequency (\%) for categorical variables. Normality of data was tested by Kolomogrov-Smirnov test. Fisher's exact test was empolyed for analysis of categorical variables, and the odds ratios (OR) with $95 \%$ confidence interval (CI) were calculated for categorical comparisons. Spearman's correlation coefficients were calculated to evaluate the relationship between the two depression questionnaires and the kappa statistics to measure the concordance between them. All test were twotailed and the statistical significance was set at $\mathrm{p}<0.05$. Statistical analysis was performed by using GraphPad InStat3.

\section{Results}

\section{Patients' charts analysis}

From the 320 patients' charts that were initially evaluated, data from the charts of subjects included in the active screening study was subsequently excluded, in order to avoid duplication of information, and these were separately analyzed. Finally, we had 287 charts analyses. Demographic data of these patients is presented in table I, which

Table I. Demographic and clinical characteristics of patients of whom the charts were evaluated retrospectively (data represents mean $\pm S D$, unless otherwise specified).

\begin{tabular}{lc}
\hline & $\begin{array}{c}\text { Patients characteristics } \\
\text { (data from medical charts) } \\
(\mathrm{n}=287)\end{array}$ \\
\hline Gender (F/M) (no/\%) & $141(49.1) / 146(50.8)$ \\
\hline Residence (urban/rural) (no/\%) & $189(65.8) / 98(34.1)$ \\
\hline Age (years) & $63.9 \pm 10.8$ \\
\hline Duration of diabetes (years) ${ }^{*}$ & $4.0(0.0-27.0)$ \\
\hline BMl (kg/m2) & $31.9 \pm 5.7$ \\
\hline Blood pressure (systolic/diastolic) $(\mathrm{mmHg})$ & $136.1 \pm 18.5 / 78.1 \pm 10.8$ \\
\hline HbA1c (\%) & $6.6 \pm 1.1$ \\
\hline Fasting blood glucose (mg/dl) & $125.1 \pm 25.1$ \\
\hline${ }^{*}$ median (min-max) &
\end{tabular}


also contains clinical data, including glycemic parameters (last available HbA1c and glycemic values were collected).

The information recorded in the 287 charts indicated that 38 patients $(13.2 \%)$ were previously diagnosed with depression, 18 (6.3\%) with anxiety (either as a separate diagnosis or combined with depression) and $7(2.4 \%)$ with cognitive dysfunction (including dementia). Out of the 38 patients with depression, $31(81.6 \%)$ were female and $7(18.4 \%)$ were male. This pointed out that the prevalence of depression was significantly higher in female compared to male subjects registered with T2D $(22.0 \%$ vs. $4.8 \%$; OR: 5.596, 95\%CI: 2.374-13.194; p<0.0001). Similarly, 14 out of 18 subjects with diagnosis of anxiety $(77.8 \%)$ were female and $4(22.2 \%)$ were men and thus, the prevalence of anxiety was also significantly higher in female patients with T2D (9.9\% vs. $2.7 \%$; OR: 3.913 , $95 \% \mathrm{CI}$ : 1.255-12.199; p: 0.01). Among the 7 patients with cognitive dysfunction, 6 were females $(85.7 \%)$ and 1 male $(14.3 \%)$ and it turned out that the prevalence of cognitive impairment was not significantly different between the two genders ( $4.3 \%$ vs $0.7 \%$, OR: $6.444,95 \%$ CI: 0.7655 - 54.255; p: 0.06).

Next, in order to verify these results, we pooled together the 287 retrospectively assessed charts with the 216 charts of subjects that were actively screened by questionnaires and thus we reviewed a total of 503 charts. Of the 503 patients with T2D, 274 (54.4\%) were women and 229 (45.5\%) were men. The diagnosis of depression was recorded in $71(14.1 \%)$ patients' charts, anxiety in 38 (7.6\%) and cognitive dysfunction in $11(2.2 \%)$ charts. When data was analyzed according to gender we practically confirmed the partial results mentioned above. The prevalence of depression was significantly higher in women than men with T2D (20.4\% vs. $6.6 \%$; OR: 3.665, 95\%CI: 2.011-6.680; $\mathrm{p}<0.0001$ ), as was the prevalence of anxiety (associated or not with depression) (10.6\% vs. $3.9 \%$; OR: $2.893,95 \% \mathrm{CI}$ : 1.340-6.248; p: 0.0061). The prevalence of recorded cognitive dysfunction was similar between genders $(2.9 \%$ vs. 1.3\%; OR: 2.266, 95\%CI: 0.5938-8.644; p: 0.35).

\section{Questionnaire results - depression}

In the active screening study we have included 216 subjects with T2D that have agreed to participate. The demographic data of these patients is presented in Table II.

The general questionnaire asked the patients if they had ever been told that they had depression. Of the 216 questioned patients, $41(19.0 \%)$ declared that they have been previously told (at some point in their lives) that they had depression and 175 (81.0\%) have not. Of those who declared that they were aware of diagnosis of depression, 36 $(87.8 \%)$ were women and $5(12.2 \%)$ were men, while of those who declared that were never diagnosed with depression, 78 (44.6\%) were men and 97 (55.4\%) were women. This indicated that significantly more women were aware of a previous diagnosis of depression (OR: 5.790, 95\%CI: 2.169-15.457; $\mathrm{p}<0.0001)$.
After administration of the two depression questionnaires, it resulted that $74(34.3 \%)$ of investigated patients with T2D had depression, as they reached the depression scores in both questionnaires (i.e. CES-D score $\geq 16$ points and PHQ-9 score $\geq 5$ points, respectively). Out of them, 16 $(21.6 \%)$ had scores indicative of major depression in both questionnaires (CES-D score $\geq 27$ and PHQ-9 score $\geq 15$ ). These basically comprised $7.4 \%$ of all 216 questioned patients with T2D. In addition, 96 (44.4\%) of patients had negative scores in both CES-D and PHQ-9 questionnaires, while the remaining 46 reached the positive scores only in one of the two questionnaires (considered by us intermediary or not conclusive results, and for the purpose of simplification of analysis, this data was pooled together with negative scores).

The analysis of the PHQ-9 questionnaire alone indicated that 114 patients $(52.8 \%)$ had scores indicative of depression: 54 (25.0\%) mild, 36 (16.7\%) moderate and 24 $(11.1 \%)$ severe depression, respectively. This might indicate an overestimation of the depression rate by administration of this single questionnaire alone (likelihood ratio (LR): 1.541, p: 0.0001). The analysis of the CES-D questionnaire only indicated that $80(37.0 \%)$ patients had scores indicative of depression: 56 (25.9\%) mild and 24 (11.1\%) severe depression (LR: 1.081, p: 0.61). It should be noted, that although numerically the same, the patients with scores of severe depression in the two questionnaires did not entirely superimpose. The Spearman coefficient of correlation was r: 0.8076 (95\%CI: 0.7538-0.8506, $\mathrm{p}<0.0001)$, indicating a very strong positive correlation between the two questionnaires, but with the recommended screening cutoffs, the agreement between the two tests was moderate (unweighted kappa: 0.531, 95\% CI: 0.434-0.628).

Analysis of the questionnaire data by gender pointed out that 56 out of 74 patients with positive scores for depres-

Table II. Demographic characteristics of patients actively evaluated by questionnaires (data represents mean $\pm S D$, unless otherwise specified).

\begin{tabular}{lc}
\hline & $\begin{array}{c}\text { Patients characteristics (screened) } \\
(\mathrm{n}=216)\end{array}$ \\
\hline Gender (F/M) (no/\%) & $133(61.5) / 83(38.4)$ \\
\hline Residence (U/R) (no/\%) & $183(84.7) / 33(15.3)$ \\
\hline Age (years) & $62.2 \pm 7.8$ \\
\hline Duration of T2D (years) ${ }^{\star}$ & $4.0(0.5-25.0)$ \\
\hline Education (no/\%): & $7(3.2)$ \\
$<8$ classes & $72(33.3)$ \\
$<12$ classes & $59(27.3)$ \\
High school & $37(17.1)$ \\
Post-secondary school & $35(16.2)$ \\
University & $6(2.8)$ \\
Post-university (master/doctoral) & \\
\hline Ethnicity (no/\%): & $161(74.5)$ \\
Romanian & $51(23.6)$ \\
Hungarian & $4(1.9)$ \\
Rroma & $154(71.3)$ \\
\hline Social status (no/\%): & $62(8.7)$ \\
Married/life-partner & \\
Never married/ widower/di- & \\
vorced &
\end{tabular}


sion were women and 18 were men. This showed again a significantly higher prevalence of depression in female than male patients with T2D ( $42.1 \%$ vs. $21.7 \%$; OR: 2.626 , 95\%CI: 1.405-4.909; p: 0.0021). However, the prevalence of severe depression was not significantly different in women compared to men $(9.0 \%$ vs. $4.8 \%$; OR: $1.959,95 \% \mathrm{CI}$ : 0.6098-6.291; p: 0.29).

When the questionnaire data was compared with data of the same patients recorded in their medical charts, it resulted that significantly more patients with $\mathrm{T} 2 \mathrm{D}$ had depression than recorded in the charts $(34.3 \%$ vs. $13.9 \%$, LR: 2.467; $\mathrm{p}<0.0001)$. This remained true when the questionnaire data was compared with data obtained from the total 503 charts (34.3\% vs. $14.1 \%$, LR: 2.427 ; $\mathrm{p}<0.0001)$.

Finally, we confronted the data from charts with data declared by patients in the general questionnaire and the scores obtained in the CES-D and PHQ-9 questionnaires. Data is presented in figure 1. Almost half of the patients (42.4\%) that were aware of the diagnosis of depression and scored positive for depression in both questionnaires never declared the diagnosis of depression and this was not noted in their medical charts. Eight out of 41 patients $(19.5 \%)$ that were told they had depression scored negative at screening questionnaires and most of them were not on medication, possibly meaning that depression was under control or that it was a temporary state. In addition, 40 $(18.5 \%)$ patients with T2D that were not aware of having depression and did not have the diagnosis recorded in their charts, had reached positive scores in both CED-D and
PHQ-9 questionnaires, with 10 of them having scores suggestive of major depression. These basically represent new or undiagnosed cases of depression.

\section{Questionnaire results - anxiety}

The results of the GAD-7 questionnaires indicated that 97 patients with T2D (44.9\%) had anxiety: 20 (9.2\% of total investigated patients) had reached major, 14 (6.5\%) moderate and $63(29.2 \%)$ minor anxiety scores, respectively.

When the questionnaire data was compared with data of the same patients recorded in their charts, it resulted that significantly more patients with T2D had anxiety than recorded in the charts (44.9\% vs. $9.3 \%$, LR: 4.850 ; $\mathrm{p}<0.0001)$. The same was observed when the questionnaire data was compared with data collected from the total 503 examined charts ( $44.9 \%$ vs. $7.6 \%$, LR: 5.944; $\mathrm{p}<0.0001$ ).

Among patients with anxiety, 64 (66.0\%) concomitantly had depression, while only 10 out of the 119 patients without anxiety (8.4\%) also had depression, indicating that anxiety is highly correlated with depression in subjects with T2D (OR: 21.139, 95\%CI: 9.767-45.751; $\mathrm{p}<0.0001)$. Similar to depression, women with T2D had significantly higher prevalence of anxiety $(51.1 \%$ vs. 34.9\%, OR: 1.948, 95\%CI: 1.107-3.428; p: 0.02).

\section{Questionnaire results - cognitive dysfunction}

After administration of the MoCA test, it resulted that 54 (25.0\%) patients had normal cognitive function scores ( $\geq 26$ points), $149(69.0 \%)$ patients had mild cognitive

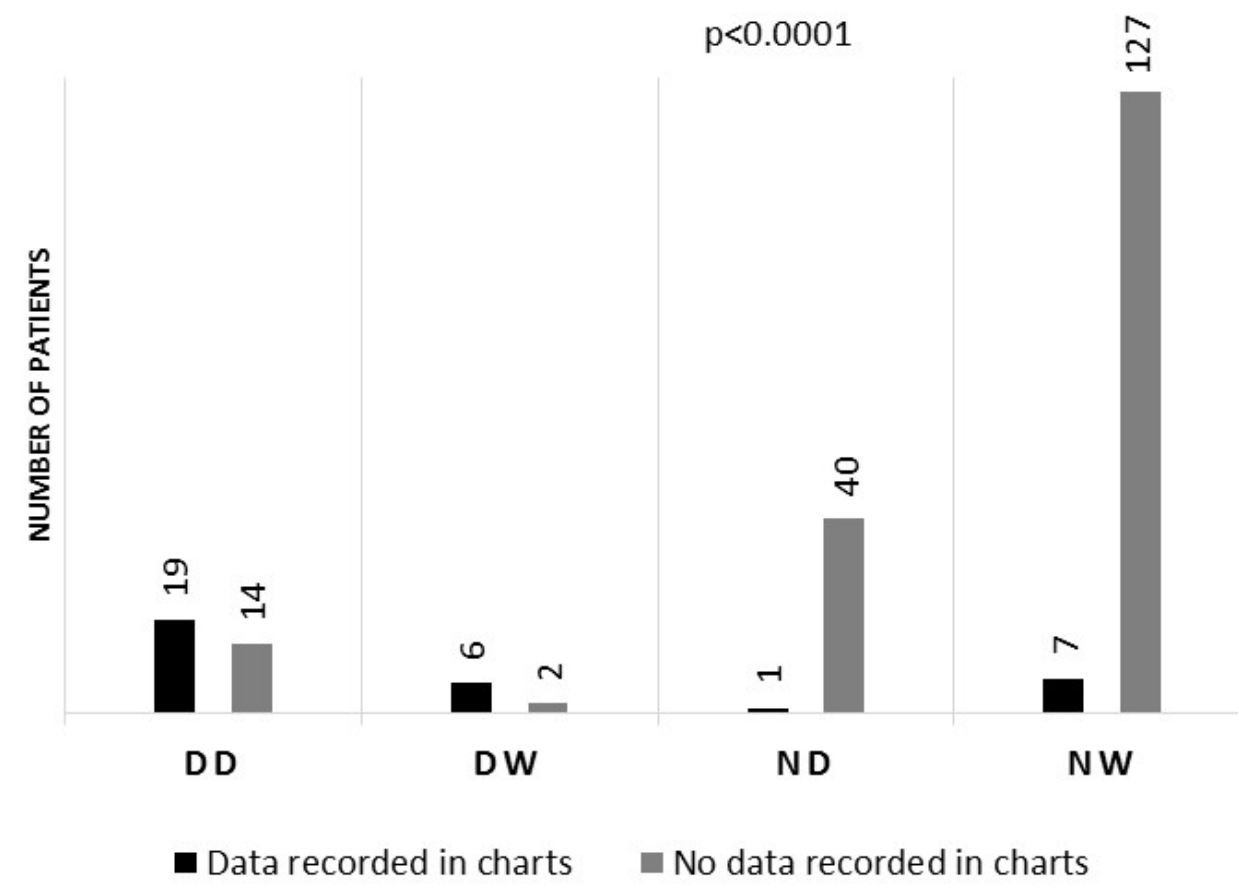

Fig. 1. Comparison of the data obtained from the active screening of depression by the CES-D and PHQ-9 questionnaires and information declared by patients in the general questionnaire with data recorded in their charts (DD: previous diagnosis of depression declared by patients and positive depression scores resulting from active screening by questionnaires; DW: previous diagnosis of depression declared by patients and negative depression scores resulting from active screening by questionnaires; ND: previous diagnosis of depression not declared by patients and positive depression scores resulting from active screening by questionnaires; NW: previous diagnosis of depression not declared by patients and negative depression scores resulting from active screening by questionnaires). 
impairment scores (17-26 points), 13 patients (6.0\%) reached scores indicative of moderate cognitive dysfunction (10-16 points) and none had $<10$ points (severe cognitive dysfunction).

When the questionnaire data was compared with that recorded in patients' charts, it turned out that significantly more patients with T2D have cognitive dysfunction than acknowledged and recorded in the charts $(75.0 \%$ vs. $1.9 \%$, LR: $40.5 ; \mathrm{p}<0.0001)$. The same was observed when the questionnaire data was compared with that recorded in the 503 examined charts $(75.0 \%$ vs. $2.2 \%$, LR: 31.438 ; $\mathrm{p}<0.0001)$. However, we assumed that only subjects with significant memory loss or cognitive impairment seek professional help, and therefore we also compared the moderate impairment scores with data in the charts. Still higher proportion of patients presented moderate cognitive dysfunction than diagnosed and recorded in the medical charts $(6.0 \%$ vs. $1.9 \%$, LR: 3.250 ; p: 0.04 and $6.0 \%$ vs. 2.2\%, LR: 2.523; p: 0.02, respectively).

We then looked at the frequency of cognitive impairment in patients with depression and anxiety compared with those without these conditions. Among depressive patients, significantly more presented mild and moderate cognitive impairment $(77.0 \%$ and $8.1 \%$, respectively), than normal cognitive scores $(14.9 \%)$, while in patients without depression, more had normal cognitive function $(30.3 \%)$, as $64.8 \%$ and $4.9 \%$ had mild and moderate dysfunction, respectively (p: 0.03) (figure 2).

Similarly, significantly more patients with different degrees of anxiety had mild or moderate cognitive dysfunction $(74.2 \%$ and $8.2 \%$, respectively) than normal cognitive scores $(17.5 \%)$, while more subjects without anxiety had normal cognitive scores $(31.1 \%)$, as $64.7 \%$ and $4.2 \%$ had mild and moderate cognitive impairment, respectively (p: 0.04) (figure 2). It should be noted though that, as mentioned above, $66 \%$ of patients with anxiety also had concomitant depression.

\section{Discussions}

To our knowledge, this is the first systematic evaluation of the prevalence of depression, anxiety and cognitive dysfunction in subjects with T2D in Romania.

We have decided to use two validated questionnaires, PHQ-9 and CES-D, both simple and quick tools for the screening of depression in clinical practice. By using only one questionnaire with currently recommended thresholds, the prevalence of depression was slightly overestimated and this was also observed in other similar studies (25, 26). We therefore consider that by concomitantly employing both questionnaires we obtained a better evaluation of depression.

In our patient population with $\mathrm{T} 2 \mathrm{D}$, regularly seen in an Outpatient Diabetes Clinic from the Central part of Romania, the prevalence of depression was high, as about a third of them (34.3\%) scored positive for depression in both questionnaires. The reported prevalence of depression in subjects with T2D varied largely in similar studies in other geographical areas, from $9.8 \%$ in Basque region and $13 \%$ in Ethiopia to $40.3 \%$ in Malesia and Nepal and $48.27 \%$ in Mexico for example, and these differences could potentially be related to methodology, variations in characteristics of the study populations (e.g. duration of diabetes, age) or simply due to genetic/ethnic backgrounds $(9,10$, 12-14). In Central and Eastern European countries, the reported rates of depression in patients with diabetes are relatively high: $30.3 \%$ in Germany, $29.7 \%$ in Poland, $28.4 \%$ in Lithuania and $22 \%$ in Croatia $(11,28-30)$. Similar to these results, the prevalence of depression in our patient population was about one in three patients. Data regarding the prevalence of depression in the general population in Romania is scarce. A report indicated that in 2008 the prevalence of major depressive episode on examination was approximately $9 \%$ and over $21 \%$ during lifetime, the prevalence being twice higher in females (31). Another study conducted between 2005 and 2007 reported much lower prevalence of major depressive episode during lifetime

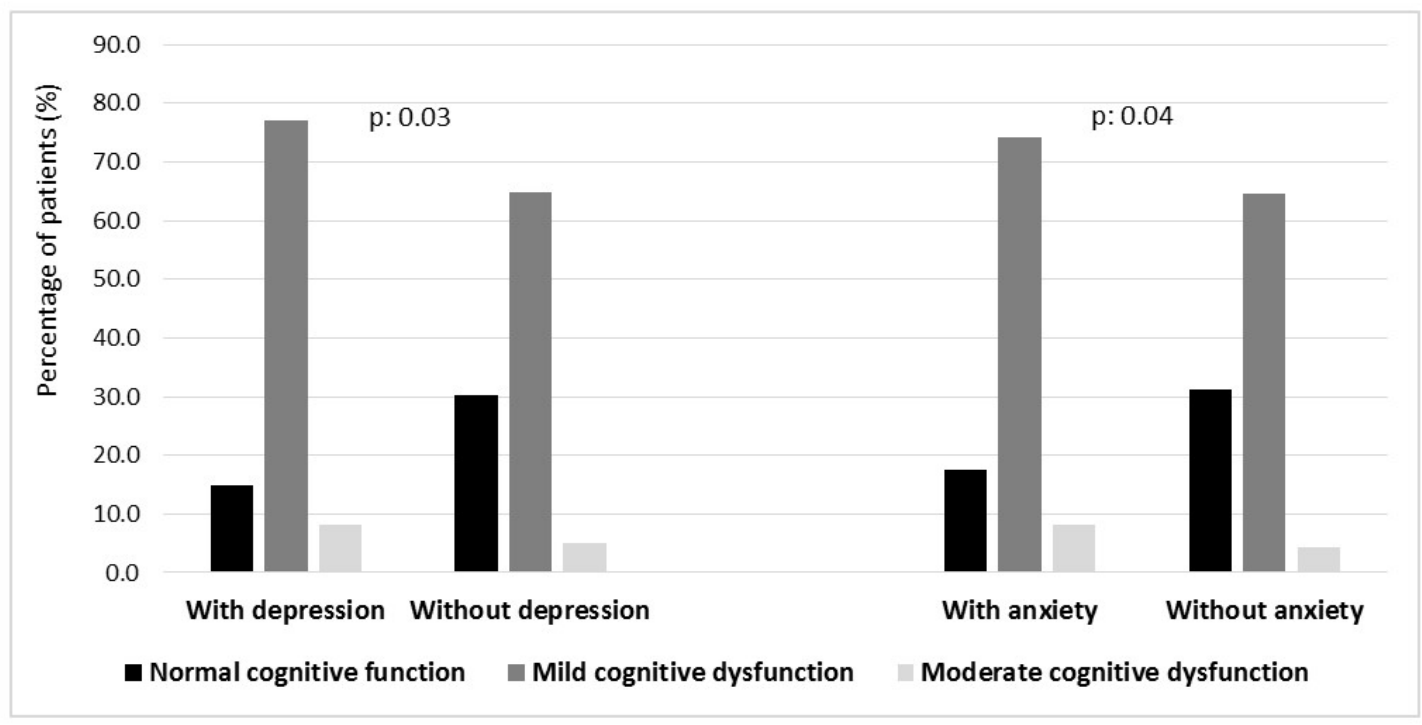

Fig. 2. Frequency of cognitive dysfunction in patients with and without depression and anxiety, respectively. 
(3.3\%), with higher rates in females (4.1\% vs. $2.5 \%)(32)$. In view of these reports, even if they are somewhat discordant, our data indicate a higher prevalence of depression in subjects with T2D compared to the general population in Romania, but a concomitant evaluation of both diabetic and non-diabetic subjects will provide a better estimation and a more meaningful comparison.

A second finding of our study was that female patients with T2D presented a frequency of depression twice higher compared with men $(42.1 \%$ vs. $21.7 \%$ with an OR: 2.626). This is in concordance with data from the general population, as well as from studies conducted in subjects with diabetes from other countries (e.g. 15.1\% females vs. $5.2 \%$ males in Basque country and $32.3 \%$ females vs. $21.8 \%$ males in Lithuania, respectively) $(9,11,31,32)$. The gender differences might be explained by hormonal or other biological factors, environmental experiences, including social demands and work burden or to psychological attributes and coping skills related to life events, but this is beyond the scope of the present work (33).

The prevalence of anxiety was even higher than that of depression: almost half of the screened T2D patients (44.9\%) had different degrees of anxiety and women had higher rates compared with men. Other studies have also indicated elevated prevalence of anxiety in subjects with T2D (e.g. 31.4\% in Malaysia, 42.4\% in Lithuania, $43.8 \%$ in Brazil, $55.1 \%$ in Mexico), with females being more affected than men (e.g. $46.8 \%$ in females vs. $34.7 \%$ in males, in Lithuania) $(10,11,34)$.

Anxiety and depression in patients with diabetes were shown to be associated with poor health behaviors and medical outcomes, including chronic complications, impaired quality of life, work disability and premature mortality $(2,4,34,35)$. It should be noted though that the relationship between the mental and physical health problems is bi-directional, as the presence of serious chronic diabetes complications, for example, might lead to anxiety or depressive symptoms. Moreover, this relationship may become cyclical, in the sense that one disease can aggravate the other: the presence of depression with/without anxiety may be associated with less self-care and adherence to diabetes management plan, which leads to poor blood glucose control and higher risk of complications, which in turn leads to increased distress, anxiety and depression.

We have also observed that the real frequency of depression and anxiety in subjects with T2D was significantly higher than recorded in their clinic charts. This may in part be due to under-reporting of the psychiatric problems, to under-evaluation or both. Out of the 216 screened patients with T2D, 19\% scored positive in both questionnaires and were not aware that they had depression. Moreover, some even suffered of severe depression, as $4.6 \%$ of the total investigated patients had reached scores suggestive of major depression and had not been previously evaluated and diagnosed with depression. Screening for the mental health disorders in subjects with diabetes is not a general prac- tice in diabetes clinics in Romania and the results of this study highlight the importance of assessing and addressing the comorbid psychiatric disorders as part of the comprehensive diabetes management plan. Early recognition and treatment of these comorbidities are likely to be associated with better self-care behaviors, better coping capabilities that can assist in improving management of diabetes and thus prevention of chronic complications.

Perhaps the most surprising finding of this study was that $69 \%$ of the patients with T2D evaluated by the MoCA test presented mild and 6.0\% moderate cognitive impairment. Moreover, in subjects with concomitant depression and anxiety the cognitive dysfunction was more frequent. A recent study indicated a similar prevalence of mild cognitive dysfunction in Japanese diabetic subjects (72\%) evaluated with the Japanese version of the MoCA test (36). Another study in older Korean subjects with T2D showed a lower prevalence of mild cognitive dysfunction $(32.7 \%)$, but the authors also used a lower threshold value for positivity ( $<23$ points) (37). It is known that diabetes is an independent risk factor for cognitive dysfunction and dementia $(38,39)$. Chronic hyperglycemia, history of repetitive moderate-to-severe hypoglycemic episodes, insulin resistance, amyloid deposition and vascular disease are factors that contribute to the occurrence of diabetes-related cognitive dysfunction $(40,41)$. The presence of comorbid major depression in patients with diabetes was shown to be associated with more than double the risk for developing dementia (42). This suggests that the evaluation and treatment of these mental conditions in patients with diabetes is important.

Out study has some limitations. First, it had a cross-sectional design, and therefore the temporal relationship between diabetes, depression, anxiety and cognitive dysfunction, respectively, as well as the change of the prevalence of these psychiatric conditions in time, could not be assessed. Secondly, the scarcity of appropriate studies evaluating the current prevalence of depression, anxiety and cognitive impairment in the general population in Romania, prevented us from performing a substantial comparison of the prevalence of these mental health conditions in diabetic vs. nondiabetic subjects in our area. Thirdly, we used psychometric scales rather than a structured interview to evaluate anxiety and depression. However, these instruments are well validated and frequently used as screening tools for psychiatric comorbidities in patients with diabetes. In addition, we have concomitantly employed two depression questionnaires, in order to avoid false positive results. Although the MoCA test is well validated and largely used worldwide, the Romanian translation does not have validation studies, as it has for many other languages. Finally, this was a single-center study with a relatively low number of evaluated subjects. However, the study population comprised both inhabitants of urban and rural areas in our county from the Central part of Romania, from most ethnic backgrounds and education levels. 
Following the results of this study we have identified several future research directions. First, it is necessary to expand the evaluation by performing a multi-center study with much higher number of T2D (and perhaps T1D) patients representative from all areas of Romania. Second, the potential mechanisms and factors (e.g. biologic, genetic, psychologic/behavioral, nutritional) associated with the high prevalence of these mental health problems in patients with diabetes should be more deeply explored. In addition, the implication of early identification and management of the psychiatric comorbidities on diabetes related-outcomes, such as blood glucose control, self-care regimens, or chronic complications and associated health care cost warrant further investigation.

The findings of this study are also relevant for clinical practice. They encourage diabetologists to pay more attention to the physiological aspects of patients with T2D, not only related to diabetes care per se, and be aware of the high prevalence of co-morbid depression, anxiety and cognitive impairment. We think that is important to screen for these conditions as they seem to be viewed as new emerging complications of diabetes. In addition, results showing increased prevalence of these mental health conditions in patients with diabetes should be considered for public health policies, as more substantial psychological/psychiatric support for them is obviously needed. Perhaps, preventive strategies to promote mental health and improve quality of life in patients with diabetes should be implemented.

\section{Conclusions}

The study showed that patients with T2D had a high prevalence of comorbid mental health problems, such as depression, anxiety and impaired cognitive function. Both depression and anxiety were significantly more frequent in women than men with diabetes. Anxiety was highly correlated to depression in these patients. These psychiatric conditions are under-evaluated and/or under-reported in patients with T2D, so an active screening is necessary.

\section{Acknowledgments}

This study was supported by an Internal Research Grant of the University of Medicine and Pharmacy of Târgu Mureş, Romania (contract number 1/23.12.2014). The authors thank Septimiu Voidăzan for his valuable comments.

\section{Conflict of interest}

There is no conflict of interest to report.

\section{References}

1. Egede LE, Ellis C. Diabetes and depression: global perspectives. Diabetes Res ClinPract. 2010;87:302-12.

2. Zhang X, Norris SL, Gregg EW, et al. Depressive symptoms and mortality among persons with and without diabetes. Am J Epidemiol 2005;161:652-60.

3. Katon W, Fan MY, Unützer J, et al. Depression and diabetes: a potentially lethal combination. J Gen Intern Med 2008;23:1571-5.

4. Egede LE, Nietert PJ, Zheng D. Depression and all-cause and coronary heart disease mortality among adults with and without diabetes. Diabetes
Care 2005;28:1339-45

5. Oladeji BD, Gureje O. The comorbidity between depression and diabetes. Curr Psychiatry Rep 2013;15:390.

6. Dash SK. Cognitive impairment and diabetes. Recent Pat Endocr Metab Immune Drug Discov 2013;7:155-65.

7. Tabák AG, Akbaraly TN, Batty GD, Kivimäki M. Depression and type 2 diabetes: a causal association? Lancet Diabetes Endocrinol 2014;2:23645

8. Roy T, Lloyd CE.Epidemiology of depression and diabetes: a systematic review. J Affect Disord 2012;142:S8-21.

9. Alonso-Morán E, Satylganova A, Orueta JF, Nuño-Solinis R. Prevalence of depression in adults with type 2 diabetes in the Basque Country: relationship with glycaemic control and health care costs. BMC Public Health 2014;14:769.

10. Ganasegeran K, Renganathan P, Manaf RA, Al-Dubai SA. Factors associated with anxiety and depression among type 2 diabetes outpatients in Malaysia: a descriptive cross-sectional single-centre study. BMJ Open 2014;4:e004794.

11. Mikaliūkštiené A, Žagminas K, Juozulynas A, et al. Prevalence and determinants of anxiety and depression symptoms in patients with type 2 diabetes in Lithuania. Med SciMonit 2014;20:182-90.

12. Niraula K, Kohrt BA, Flora MS, et al. Prevalence of depression and associated risk factors among persons with type-2 diabetes mellitus without a prior psychiatric history: a cross-sectional study in clinical settings in urban Nepal. BMC Psychiatry 2013;13:309.

13. Dejenie Habtewold T, Radie YT, Sharew NT. Prevalence of Depression among Type 2 Diabetic Outpatients in Black Lion General Specialized Hospital, Addis Ababa, Ethiopia. Depress Res Treat 2015;2015:184902.

14. Tovilla-Zárate C, Juárez-Rojop I, Peralta Jimenez Y, et al. Prevalence of anxiety and depression among outpatients with type 2 diabetes in the Mexican population. PLoS One. 2012;7:e36887.

15. Li C, Ford ES, Zhao G, et al. Prevalence and correlates of undiagnosed depression among U.S. adults with diabetes: the Behavioral Risk Factor Surveillance System, 2006. Diabetes Res ClinPract. 2009;83:268-79.

16. Culman M, Guja C, Mihai A, Serafinceanu C. Depression screening and risk profile in diabetic patients. Rom J Leg Med 2013;21:197-200.

17. American Diabetes Association.Standards of Medical Care in Diabetes. Diabetes Care 2015;38(Suppl.1):S41-S48.

18. Radloff LS. The CES-D Scale: A Self-Report Depression Scale for Research in the General Population. Applied Psychological Measurement 1977;1:385-401.

19. Hermanns N, Caputo S, Dzida G, et al. Screening, evaluation and management of depression in people with diabetes in primary care. Prim Care Diabetes 2013;7:1-10.

20. Holt RI, de Groot M, Golden SH. Diabetes and depression. CurrDiab Rep 2014;14:491.

21. Zich JM, Attkisson CC, Greenfield TK. Screening for depression in primary care clinics: the CES-D and the BDI. Int J Psychiatry Med 1990;20:259-77.

22. Geisser ME, Roth RS, Robinson ME. Assessing depression among persons with chronic pain using the Center for Epidemiological StudiesDepression Scale and the Beck Depression Inventory: a comparative analysis. Clin J Pain 1997;13:163-70.

23. Kroenke K, Spitzer RL, Williams JB. The PHQ-9: validity of a brief depression severity measure. J Gen Intern Med 2001;16:606-13.

24. Spitzer RL, Kroenke K, Williams JB, Löwe B. A brief measure for assessing generalized anxiety disorder: the GAD-7. Arch Intern Med 2006;166:1092-7.

25. Nasreddine ZS, Phillips NA, Bédirian V, et al. The Montreal Cognitive Assessment, MoCA: A Brief Screening Tool For Mild Cognitive Impairment. J Am GeriatrSoc2005;53:695-699

26. Reddy P, Philpot B, Ford D, Dunbar JA. Identification of depression in diabetes: the efficacy of PHQ-9 and HADS-D. Br J Gen Pract. 2010;60:e239-45.

27. Twist K, Stahl D, Amiel SA, et al. Comparison of depressive symptoms in type 2 diabetes using a two-stage survey design. Psychosom Med. 2013;75:791-7

28. Gorska-Ciebiada M, Saryusz-Wolska M, Ciebiada M, Loba J. Mild cognitive impairment and depressive symptoms in elderly patients with diabetes: prevalence, risk factors, and comorbidity. J Diabetes Res. 2014;2014:179648.

29. Pibernik-Okanovic M, Peros K, Szabo S, Begic D, Metelko Z. Depression in Croatian Type 2 diabetic patients: prevalence and risk factors. A Croatian survey from the European Depression in Diabetes (EDID) Research Consortium.Diabet Med. 2005;22:942-5.

30. Jacob L, Kostev K. Prevalence of depression in type 2 diabetes patients in German primary care practices. Journal of Diabetes and Its 
Complications.In Press. Published onlineDOl:http://dx.doi.org/10.1016/j. jdiacomp.2015.12.013

31. Patriche D, Filip I, Tănase C. Epidemiology of depression. Revista Medicală Română. 2015;3:260-262

32. Florescu S, Popovici G, Ciutan M, Ladea M, Sorel E. Management in sănătate. 2010;2:28-37.

33. Kessler RC. Epidemiology of women and depression.J Affect Disord. 2003; 74:5-13.

34. de Ornelas Maia AC, Braga Ade A, Paes F, et al. Comorbidity of depression and anxiety: association with poor quality of life in type 1 and 2 diabetic patients. ClinPractEpidemiolMent Health. 2013;9:136-41

35. Khuwaja AK, Lalani S, Dhanani R, et al. Anxiety and depression among outpatients with type 2 diabetes: A multi-centre study of prevalence and associated factors. DiabetolMetabSyndr. 2010;2:72.

36. Mori $\mathrm{Y}$, Futamura A, Murakami $\mathrm{H}$, et al. Increased detection of mild cognitive impairment with type 2 diabetes mellitus using the Japanese version of the Montreal Cognitive Assessment: A pilot study. Neurology and Clinical Neuroscience. 2015;3:89-93

37. Lee YJ, Kang HM, Kim NK, et al. Factors associated for mild cognitive impairment in older korean adults with type 2 diabetes mellitus. Diabetes Metab J. 2014;38:150-7.

38. Reijmer YD, van den Berg E, Ruis C, Kappelle LJ, Biessels GJ. Cognitive dysfunction in patients with type 2 diabetes. Diabetes Metab Res Rev. 2010;26:507-19.

39. Mayeda ER, Whitmer RA, Yaffe K. Diabetes and cognition. ClinGeriatr Med. 2015;31:101-15

40. McCrimmon RJ, Ryan CM, Frier BM. Diabetes and cognitive dysfunction. Lancet. 2012;379:2291-9.

41. Kodl CT, Seaquist ER.Cognitive dysfunction and diabetes mellitus. Endocr Rev. 2008;29:494-511.

42. Katon WJ, Lin EH, Williams LH, et al. Comorbid depression is associated with an increased risk of dementia diagnosis in patients with diabetes: a prospective cohort study. J Gen Intern Med. 2010;25:423-9 